\title{
BIBECHANA
}

A Multidisciplinary Journal of Science, Technology and Mathematics

ISSN 2091-0762 (Print), 2382-5340 (Online)

Journal homepage: http://nepjol.info/index.php/BIBECHANA

Publisher: Research Council of Science and Technology, Biratnagar, Nepal

\section{General survey on avifaunal composition of Betana wetland, Morang, Province number 1, Eastern Nepal}

\author{
Milan Kharel \\ Department of Zoology, Central Campus of Technology, Tribhuvan University, Dharan \\ E-mail id: milankharel2000@gmail.com
}

Article history: Received 22 September, 2018; Accepted 2 November, 2018

DOI: http://dx.doi.org/10.3126/bibechana.v16i0.21570

This work is licensed under the Creative Commons CC BY-NC License. https://creativecommons.org/licenses/by$\mathrm{nc} / 4.0 /$

The present study conducted in Betana wetland, Morang, Province number 1, Nepal. The study based on the field survey in order to find out the avifaunal composition. In the present study, total 320 individuals of birds belonging 49 species, 30 families and 15 orders reported from Betana wetland based on field observation. The highest proportions of individuals recorded from the sampling station F1 forest area was $35 \%(\mathrm{n}=112)$. The highest number of species $(\mathrm{n}=15)$ recorded belong to the order Passeriformes with nine families, followed by Coraciformes with three families and four species then Pelicaniformes with two families and six species. Of them, two species viz. Grey-headed fish eagle (Icthyophaga humilis) and Lesser Adjutant Stork (Leptoptilos javanicus ) are kept under near threatened (NT) and vulnerable (VU) category of IUCN Red List of threatened species. About $70 \%$ of total bird recorded was resident type and about $35 \%$ of total recorded bird species common in abundance. The species richness and abundance of avifauna found higher in winter season than summer. Compared to the previous study, birds belonging to four more families and five more orders recorded this time. The Shannon-Weiner diversity index and species evenness of avifauna from the study area found 1.332 and 0.789 respectively.

Keywords: Betana; avifauna; wetland; Icthyophaga humilis; Leptoptilos javanicus.

\section{Introduction}

After forest, more than a quarter (27\%) of Nepal's nationally threatened birds inhabits in wetlands [1]. Out of 886 species of Bird species recorded so far in Nepal, about 42 species recorded in Nepal are listed in IUCN Red List of globally threatened birds, 35 globally near threatened and 167 species are nationally threatened [2]. More than 230 species of birds found to be wetland-dependent in Nepal [3]. Wetlands are among the most productive ecosystem in the world. The wetlands of Nepal well known for their unusually 
rich biodiversity. They occupy approximately five percent of the total area of Nepal in the form of rivers, stream, lakes, reservoirs, village ponds, paddy fields, marsh and swampland. There are over 405 wetland areas in Nepal from the Terai to the Himalayas. The loss of diversity of the water birds reduces the natural resource base of the country. Wetlands are one of the most threatened habitats because of their vulnerability and attractiveness for development [4]. Betana wetland is an important recreational destination with great potential of wildlife including bird diversity. Now this area has established as a picnic spot and other recreational activities that have created lots of problem on habitat, breeding and feeding activities of birds. Firewood collection and grazing are the prominent human-induced disturbances of this area. The forest area of wetland dominated by Sal (Shorea robusta) followed by Khair-Sissoo (Acacia catechu-Dalbergia sissoo) and mixed forest. Grassland flora consisted of a combination of wetland herbs and moist grass species that are commonly composed of Imperata cylindrica, Cyperus papyrus, Digitariya ciliaris, Bulbostylis barabata, Erasgrostis tentella, Cyperus totundus, Polygonum spp.etc [5]. Data on the avifaunal composition of this area is not adequate to assess conservation needs. Only a little information from records of bird watchers, nature guides etc. are available. Hence, the present study was essential to provide baseline data on avifaunal composition for the proper conservation and management initiatives.

\section{Study area}

Betana wetland is a freshwater pond situated between $26.659106^{\circ} \mathrm{N}$ to $87.428814^{\circ} \mathrm{E}$ and $26.662894^{\circ} \mathrm{N}$ to $87.434018^{\circ} \mathrm{E}$ at an elevation of $123 \mathrm{~m} \mathrm{msl}$, covering 5.5 ha area in Belbari municipality of Morang district. It is one kilometer far from the Belbari Bazar in the east. The wetland area remains surrounded by Sal forest from east, north and west sides whereas Mahendra highway lies adjacent on its south. The depth of the pond varies from 0.5 to $1.5 \mathrm{~m}$ in the dry season and 1 to $2.5 \mathrm{~m}$ in monsoon season [6]. The study area experiences a tropical monsoon climate with winter, summer and rainy seasons in a year. The soil is alluvial type and the average annual temperature of $24.6{ }^{\circ} \mathrm{C}$. The average rainfall is $2256 \mathrm{~mm}$ per year and about $90 \%$ of rainfall occurs within three months of monsoon seasons (June-August) [7].

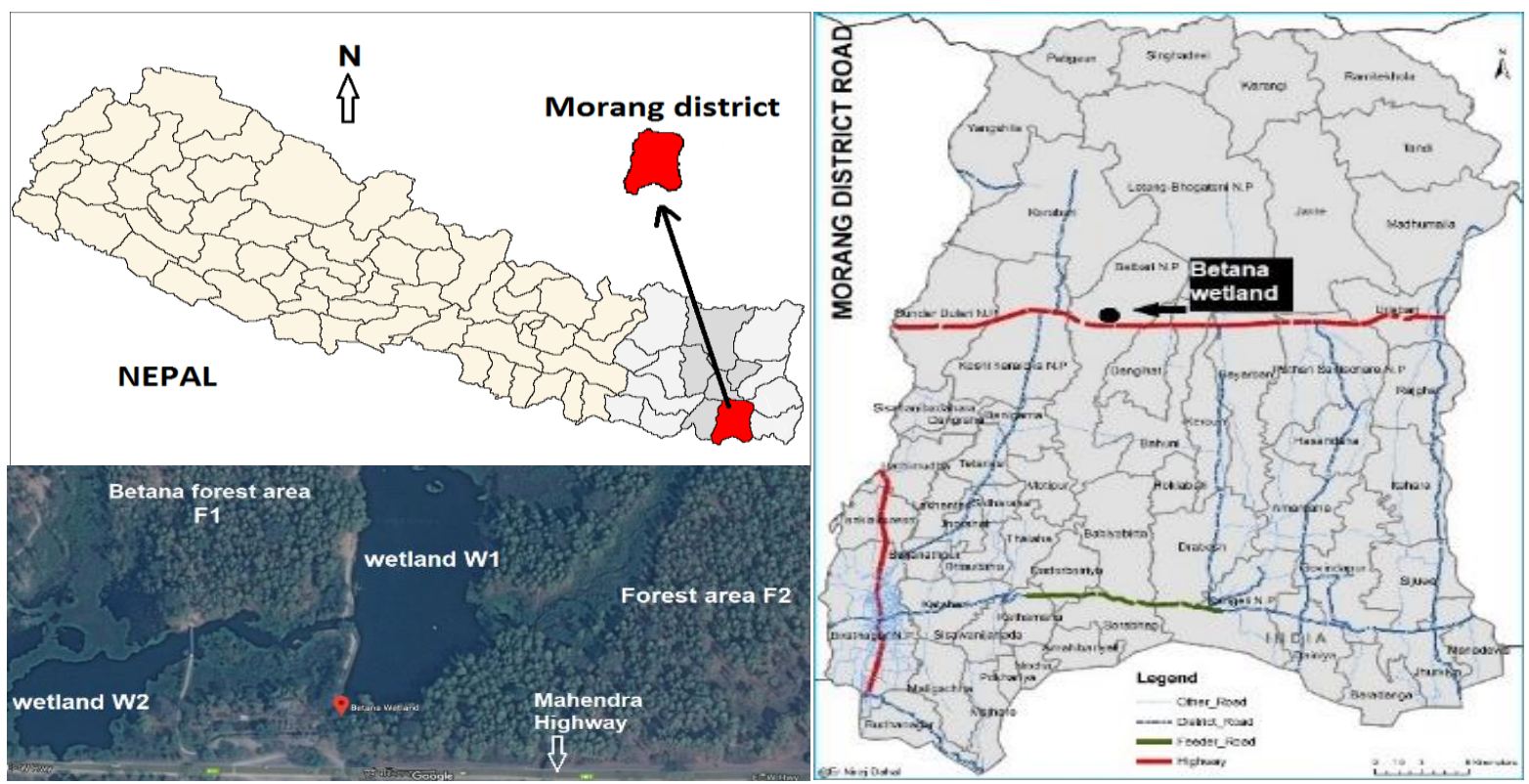

Fig.1: Map of study the site. 


\section{Material and methods}

Study of the avifaunal composition of Betana wetland carried out for six months from February 2017 to July 2017. Therefore, the birds of summer, winter and monsoon season observed within a short field visit period. Regular visits of the study site done almost once a week on every Saturday morning between $7-10$ am and 4-6 pm in the evening in order to know the avifaunal composition. The bird census was done by applying the Point Count Survey Method within the radius of 50m [8]. In the direct count method, counts performed in the four sampling stations (F1, F2, W1 and W2) repeatedly. To prevent overlapping, the total number of individuals counted only for every two or three species during the first sightings of field visits. Some birds photographed with the help of Canon Powershot SX520 HS 42x 24-1008mm 16 MP optical zoom digital camera. The primary data collected by direct observation of species with the help of Bushnell $\mathrm{H} 2 \mathrm{O}$ Waterproof/Fogproof Prism Binocular $10 \times 42 \mathrm{~mm}$. While secondary data collected by the help of a questionnaire, reviewing literature such as journals, articles, proceedings and books. The geographical coordinates were taken by using Garmin eTrex 10 Worldwide Handheld GPS navigator. The identification of birds done by direct observation method. Birds were observed within transect of $100 \mathrm{~m}$ and identified with the help of field guide books of Ali and Ripley [9], Fleming et al. [10], Shrestha [3] and Grimmett [1], Grimmett et.,al.[11] and Pradhan [12]. Photograph of unidentified species identified with the help of subject expertise of Post-graduate campus, Biratnagar. For the birds, which are shy and not observed directly, the call count method employed for their identification. The study area divided into four pockets (Map1). Bird observation done at these four pockets viz. the forest area F1 (west) and forest area F2 (east), wetland area W1 (east) and wetland area W2 (west) of Betana wetland area. Four line transects were set along the two forests sampling areas (F1 and F2) and two wetland areas (W1 and W2). Each pocket used as the reference points for the point count method. Observations of birds carried out on each $50 \mathrm{~m}$ radius of each pocket.

The collected data represented in the tabular form by using Microsoft Excel. Shannon-Weiner diversity index and species evenness calculated by using by using SPSS software version v23. Shannon-Weiner diversity index $(\mathrm{H})$ is an index that commonly used to characterize species diversity in a community. It calculated by the following function:

$$
H=-\sum[(p i) \times \ln (p i)]
$$

where,

$$
\mathrm{pi}=\text { proportion of total sample represented by species } \mathrm{i}
$$

$\mathrm{S}=$ species richness or number of different species in a given area

$\mathrm{E}=$ Evenness $=\frac{\mathrm{H}}{\ln (\mathrm{S})}$

\section{Results}

In the present study, 49 species of birds belonging to 30 families and 15 orders reported from Betana wetland based on field observation (Table 1). The highest number of species $(n=15)$ recorded belong to the order Passeriformes with nine families. The total number of individuals of birds in all four sampling stations were 320. The highest number of individuals recorded from the sampling station F1 forest area was $35 \%(n=112)$. Similarly, 27\% $(n=89)$ from $\mathrm{W} 1,23 \%(n=76)$ from F2 and 13\% $(n=43)$ from W2. The Shannon-Weiner diversity index of avifauna at four sampling stations found 1.334 and that of species evenness found to be 0.789 .

Similarly, the rest of the orders in terms of species richness recorded as Coraciiformes ( 3 families and 4 species). Pelicaniformes ( 2 families and 6 species), Accipitriformes ( 2 families and 4 species). Piciformes 
(2 families and 2 species), Charadriformes (2 families and 2 species), Anseriformes (2 families and 2 species), Strigiformes (one family and 3 species), Columbiformes (one family and 3 species), Cululiformes (one family and 2 species), Ciconiformes (one family and 2 species) and the orders representing one family and one species were Suliformes, Gruiformes and Psittaciformes. Out of 49 total species, 34 species $(69.38 \%)$ of birds were of the resident type, 9 species $(18.36 \%)$ were winter visitor and 6 species (12.24\%) were summer visitors (Fig 3). Of them, 34.69\% species $(n=17)$ were common, 24.48 $\%$ species $(\mathrm{n}=12)$ of birds were common, $22.44 \%(\mathrm{n}=11)$ species were occasional and $18.36 \%(\mathrm{n}=9)$ species were uncommon (Fig 4). The number of bird species were recorded from the sampling area were $\mathrm{F} 1=32.65 \%(\mathrm{n}=17), \mathrm{F} 2=34.69 \%(17), \mathrm{W} 1=20.40 \%(\mathrm{n}=10)$ and $\mathrm{W} 2=12.24 \%(\mathrm{n}=06)$ respectively (Fig $5)$.

Table 1: Avifaunal composition of Betana wetland.

\begin{tabular}{|c|c|c|c|c|c|c|c|c|}
\hline SN & Family & Common name & Scientific name & $\begin{array}{l}\text { Local } \\
\text { name }\end{array}$ & SS & $\mathbf{A B}$ & IUCN & HL \\
\hline \multicolumn{9}{|c|}{ ORDER- ACCIPITRIFORMES } \\
\hline 1 & Accipitridae & Black kite & $\begin{array}{l}\text { Milvus migrans } \\
\text { (Boddaert,1783) }\end{array}$ & Kalocheel & WV & $\mathrm{UC}$ & LC & F1 \\
\hline 2 & Accipitridae & $\begin{array}{l}\text { Grey-headed } \\
\text { Fish eagle }\end{array}$ & $\begin{array}{c}\text { Icthyophaga } \\
\text { humilis }^{\text {a }} \text { (Muller, S } \\
\text { \& Schlegel, 1841) }\end{array}$ & Machhakul & $\mathrm{R}$ & $\mathrm{O}$ & VU & W2 \\
\hline 3 & Pandionidae & Osprey & $\begin{array}{c}\text { Pandion haliaetus } \\
\text { (Linnaeus,1758) }\end{array}$ & $\begin{array}{l}\text { Malaha } \\
\text { cheel }\end{array}$ & WV & $\mathrm{UC}$ & $\mathrm{LC}$ & F2 \\
\hline 4 & Accipitridae & $\begin{array}{l}\text { Crested Serpent } \\
\text { Eagle }\end{array}$ & $\begin{array}{l}\text { Spilornis cheela } \\
\text { (Latham,1790) }\end{array}$ & Kakakul & $\mathrm{R}$ & $\mathrm{UC}$ & $\mathrm{LC}$ & $\mathrm{W} 1$ \\
\hline \multicolumn{9}{|c|}{ ANSERIFORMES } \\
\hline 5 & Anatidae & CommonTeal & $\begin{array}{c}\text { Anas crecca } \\
\text { (Linnaeus,1758) }\end{array}$ & Vijula Gairi & WV & $\mathrm{C}$ & LC & $\mathrm{F} 2$ \\
\hline 6 & $\begin{array}{l}\text { Dendroygnida } \\
\text { e }\end{array}$ & $\begin{array}{l}\text { Lesser Whistling } \\
\text { Duck }\end{array}$ & $\begin{array}{c}\text { Dendrocygna } \\
\text { javanica (Horsfield, } \\
1821)\end{array}$ & Silsile & WV & $\mathrm{UC}$ & $\mathrm{LC}$ & W1 \\
\hline \multicolumn{9}{|c|}{ BUCEROTIFORMES } \\
\hline 7 & Upupidae & $\begin{array}{l}\text { Common } \\
\text { Hoopoe }\end{array}$ & $\begin{array}{c}\text { Uрира ерорs } \\
\text { (Linnaeus,1758) }\end{array}$ & Fafre chara & $\mathrm{R}$ & FC & $\overline{\mathrm{LC}}$ & $\bar{F} 2$ \\
\hline \multicolumn{9}{|c|}{ CHARADRIIFORMES } \\
\hline 8 & Charadriidae & $\begin{array}{l}\text { Red- wattled } \\
\text { Lapwig }\end{array}$ & $\begin{array}{l}\text { Vanellus indicus } \\
\text { (Boddaert,1783) }\end{array}$ & Huttityaun & $\mathrm{R}$ & $\mathrm{C}$ & $\mathrm{LC}$ & W2 \\
\hline 9 & Jacanidae & $\begin{array}{l}\text { Bronze-winged } \\
\text { Jacana }\end{array}$ & $\begin{array}{c}\text { Metopidius indicus } \\
\text { (Latham, 1790) }\end{array}$ & Lama aunle & $\mathrm{R}$ & FC & LC & W1 \\
\hline
\end{tabular}




\begin{tabular}{|c|c|c|c|c|c|c|c|c|}
\hline SN & Family & Common name & Scientific name & $\begin{array}{l}\text { Local } \\
\text { name }\end{array}$ & SS & $\mathbf{A B}$ & IUCN & HL \\
\hline \multicolumn{9}{|c|}{ CICONIIFORMES } \\
\hline 10 & Ciconiidae & Lesser Adjutant & $\begin{array}{c}\text { Leptoptilos } \\
\text { javanicus }^{b} \\
\text { (Horsfield,1821) }\end{array}$ & $\begin{array}{l}\text { Bhundifor } \\
\text { garud }\end{array}$ & $\mathrm{R}$ & $\mathrm{O}$ & VU & W1 \\
\hline 11 & Ciconiidae & Asian Openbill & $\begin{array}{c}\text { Anastomus oscitans } \\
\text { (Boddaert,1783) }\end{array}$ & $\begin{array}{l}\text { Ghungifor } \\
\text { garud }\end{array}$ & SV & $\mathrm{UC}$ & LC & W1 \\
\hline \multicolumn{9}{|c|}{ COLUMBIFORMES } \\
\hline 12 & Columbidae & Rock Pigeon & $\begin{array}{l}\text { Columba livia } \\
\text { (Gmelin, } 1789\end{array}$ & Parewa & $\mathrm{R}$ & FC & $\mathrm{LC}$ & F1 \\
\hline 13 & Columbidae & Spotted dove & $\begin{array}{c}\text { Streptopelia } \\
\text { chinensis (Scopoli, } \\
1786)\end{array}$ & $\begin{array}{l}\text { Kurle } \\
\text { dhukur }\end{array}$ & $\mathrm{R}$ & $\mathrm{FC}$ & & F1 \\
\hline 14 & Columbidae & $\begin{array}{l}\text { Eurasian } \\
\text { Collared Dove }\end{array}$ & $\begin{array}{c}\text { Streptopelia } \\
\text { decaocto } \\
\text { (Frivaldszky, 1838) }\end{array}$ & $\begin{array}{l}\text { Kanthe } \\
\text { dhukur }\end{array}$ & $\mathrm{R}$ & $\mathrm{FC}$ & $\mathrm{LC}$ & F1 \\
\hline \multicolumn{9}{|c|}{ CORACIIFORMES } \\
\hline 15 & Alcedinidae & $\begin{array}{l}\text { Stork-billed } \\
\text { Kingfisher }\end{array}$ & $\begin{array}{l}\text { Halcyon capinsis } \\
\text { (Linnaeus, } 1766 \text { ) }\end{array}$ & $\begin{array}{l}\text { Thulomatik } \\
\text { ore }\end{array}$ & $\mathrm{R}$ & $\mathrm{O}$ & $\mathrm{LC}$ & F1 \\
\hline 16 & Coraciidae & Dollarbird & $\begin{array}{c}\text { Eurystomus } \\
\text { orientalis (Linnaeus, } \\
1766)\end{array}$ & $\begin{array}{l}\text { Lal chuche } \\
\text { theuwa }\end{array}$ & SV & $\mathrm{O}$ & LC & $\mathrm{F} 2$ \\
\hline 17 & Alcedinidae & $\begin{array}{l}\text { White-throated } \\
\text { Kingfisher }\end{array}$ & $\begin{array}{l}\text { Halcyon smyrnensis } \\
\text { (Linnaeus, } \underline{1758)}\end{array}$ & $\begin{array}{l}\text { Setokanthe } \\
\text { matikore }\end{array}$ & $\mathrm{R}$ & $\mathrm{O}$ & $\mathrm{LC}$ & W2 \\
\hline 18 & Meropidae & $\begin{array}{l}\text { Chestnut-headed } \\
\text { Bee-eater }\end{array}$ & $\begin{array}{c}\text { Merops } \\
\text { leschenaultia } \\
\text { (Linnaeus, 1758) }\end{array}$ & $\begin{array}{l}\text { Katus tauke } \\
\text { Murali } \\
\text { chara }\end{array}$ & SV & $\mathrm{UC}$ & $\mathrm{LC}$ & $\mathrm{F} 2$ \\
\hline \multicolumn{9}{|c|}{ CUCULIFORMES } \\
\hline 19 & Cuculidae & Indian Cuckoo & $\begin{array}{c}\text { Cuculus micropterus } \\
\text { (Gould, 1838) }\end{array}$ & $\begin{array}{l}\text { Kaphal } \\
\text { pakyo }\end{array}$ & SV & $\mathrm{O}$ & $\mathrm{LC}$ & $\mathrm{F} 2$ \\
\hline 20 & Cuculidae & Greater Couckal & $\begin{array}{c}\text { Centropus sinensis } \\
\text { (Stephens, 1815) }\end{array}$ & $\begin{array}{l}\text { Dhade } \\
\text { gokul }\end{array}$ & WV & $\mathrm{C}$ & $\mathrm{LC}$ & $\mathrm{F} 2$ \\
\hline \multicolumn{9}{|c|}{ GRUIFORMES } \\
\hline 21 & Rallidae & $\begin{array}{l}\text { White-brested } \\
\text { Waterhen }\end{array}$ & $\begin{array}{c}\text { Amaurornis } \\
\text { phoenicurus } \\
\text { (Pennant, 1769) }\end{array}$ & $\begin{array}{l}\text { Sim } \\
\text { kukhura }\end{array}$ & $\mathrm{R}$ & FC & LC & W2 \\
\hline
\end{tabular}




\begin{tabular}{|c|c|c|c|c|c|c|c|c|}
\hline SN & Family & Common name & Scientific name & $\begin{array}{l}\text { Local } \\
\text { name }\end{array}$ & SS & $\mathbf{A B}$ & IUCN & HL \\
\hline \multicolumn{9}{|c|}{ PASSERIFORMES } \\
\hline 22 & Corvidae & $\begin{array}{l}\text { Large-billed } \\
\text { Crow }\end{array}$ & $\begin{array}{c}\text { Corvus } \\
\text { macrorhynchos } \\
\text { (Wagler,1827) }\end{array}$ & Kalokag & $\mathrm{R}$ & $\mathrm{C}$ & LC & F1 \\
\hline 23 & Corvidae & Rufous Treepie & $\begin{array}{c}\text { Dendrocitta } \\
\text { vagabunda } \\
\text { (Latham,1790) }\end{array}$ & Kokale & $\mathrm{R}$ & $\mathrm{O}$ & LC & F1 \\
\hline 24 & Corvidae & House Crow & $\begin{array}{l}\text { Corvus splendens } \\
\text { (Vieillot, 1817) }\end{array}$ & Gharkag & $\mathrm{R}$ & $\mathrm{FC}$ & LC & F1 \\
\hline 25 & Dicuridae & Black Drongo & $\begin{array}{c}\text { Dicrurus } \\
\text { macrocercus } \\
\text { (Vieillot, 1817) }\end{array}$ & Kalochibe & $\mathrm{R}$ & $\mathrm{FC}$ & LC & $\mathrm{F} 2$ \\
\hline 26 & Dicuridae & $\begin{array}{ll}\text { Crow billed } \\
\text { Drongo }\end{array}$ & $\begin{array}{c}\text { Dicrurus annectans } \\
\text { (Hodgson,1838) }\end{array}$ & $\begin{array}{l}\text { Kagthude } \\
\text { chibe }\end{array}$ & SV & FC & LC & F1 \\
\hline 27 & Dicuridae & $\begin{array}{l}\text { Geater Racket- } \\
\text { tailed Drongo }\end{array}$ & $\begin{array}{c}\text { Dicrurus paradiseus } \\
\text { (Linnaeus, 1766) }\end{array}$ & $\begin{array}{l}\text { Bhimraj } \\
\text { chibe }\end{array}$ & $\mathrm{R}$ & $\mathrm{C}$ & LC & $\mathrm{F} 2$ \\
\hline 28 & Oriolidae & $\begin{array}{l}\text { Golden } \\
\text { Oriole }\end{array}$ & $\begin{array}{l}\text { Oriolus oriolus } \\
\text { (Linnaeus, } 1758 \text { ) }\end{array}$ & $\begin{array}{l}\text { Gajale } \\
\text { sunchari }\end{array}$ & SV & UC & LC & $\mathrm{F} 2$ \\
\hline 29 & Oriolidae & $\begin{array}{l}\text { Black- headed } \\
\text { Oriole }\end{array}$ & $\begin{array}{c}\text { Oriolus xanthornus } \\
\text { (Linnaeus, 1758) }\end{array}$ & $\begin{array}{l}\text { Kalotauke } \\
\text { sunchari }\end{array}$ & $\mathrm{R}$ & $\mathrm{UC}$ & $\mathrm{LC}$ & $\mathrm{F} 2$ \\
\hline 30 & Sturnidae & Common Myna & $\begin{array}{c}\text { Acridotheres tristis } \\
\text { (Linnaeus, 1766) }\end{array}$ & Dangrerupi & $\mathrm{R}$ & $\mathrm{FC}$ & LC & F1 \\
\hline 31 & Sturnidae & $\begin{array}{ll}\text { Asian } & \text { Pied } \\
\text { Starling } & \end{array}$ & $\begin{array}{l}\text { Gracupica contra } \\
\text { (Linnaeus, 1758) }\end{array}$ & $\begin{array}{l}\text { Danger } \\
\text { saraun }\end{array}$ & $\mathrm{R}$ & $\mathrm{FC}$ & LC & $\mathrm{F} 1$ \\
\hline 32 & Laniidae & $\begin{array}{l}\text { Long-tailed } \\
\text { Shrike }\end{array}$ & $\begin{array}{l}\text { Lanius schach } \\
\text { (Linnaeus, 1758) }\end{array}$ & Bhadrai & WV & UC & LC & $\mathrm{F} 2$ \\
\hline 33 & Passeridae & House Sparrow & $\begin{array}{c}\text { Passer domesticus } \\
\text { (Linnaeus, 1758) }\end{array}$ & Bhagera & $\mathrm{R}$ & $\mathrm{FC}$ & LC & F1 \\
\hline 34 & Pycnonotidae & $\begin{array}{l}\text { Red-vented } \\
\text { Bulbul }\end{array}$ & $\begin{array}{l}\text { Pycnonotus cafer } \\
\text { (Linnaeus, 1766) }\end{array}$ & Jureli & $\mathrm{R}$ & $\mathrm{FC}$ & LC & F1 \\
\hline 35 & Muscicapidae & $\begin{array}{l}\text { Oriental Magpie } \\
\text { Robin }\end{array}$ & $\begin{array}{c}\text { Copsychus saularis } \\
\text { (Linnaeus, 1758) }\end{array}$ & $\begin{array}{l}\text { Dhobi } \\
\text { chara }\end{array}$ & $\mathrm{R}$ & $\mathrm{FC}$ & $\mathrm{LC}$ & F1 \\
\hline 36 & Leiothrichidae & Jungle Babbler & $\begin{array}{c}\text { Turdoides striata } \\
\text { (Dumont, 1823) }\end{array}$ & $\begin{array}{l}\text { Bagale } \\
\text { vyakur }\end{array}$ & $\mathrm{R}$ & $\mathrm{C}$ & $\mathrm{LC}$ & $\mathrm{F} 2$ \\
\hline
\end{tabular}




\begin{tabular}{|c|c|c|c|c|c|c|c|c|}
\hline SN & Family & Common name & Scientific name & $\begin{array}{l}\text { Local } \\
\text { name }\end{array}$ & SS & $\mathbf{A B}$ & IUCN & HL \\
\hline \multicolumn{9}{|c|}{ PELICANIFORMES } \\
\hline 37 & Ardeidae & $\begin{array}{l}\text { Indian Pond } \\
\text { Heron }\end{array}$ & $\begin{array}{c}\text { Ardeola grayii } \\
\text { (Sykes, 1832) }\end{array}$ & $\begin{array}{l}\text { Askote } \\
\text { bakulla }\end{array}$ & $\mathrm{R}$ & $\mathrm{FC}$ & LC & $\mathrm{W} 2$ \\
\hline 38 & Ardeidae & Purple Heron & $\begin{array}{l}\text { Ardea purpurea } \\
\text { (Linnaeus,1766) }\end{array}$ & $\begin{array}{l}\text { Dhyani } \\
\text { bakulla }\end{array}$ & WV & $\mathrm{O}$ & LC & $\mathrm{W} 1$ \\
\hline 39 & Ardeidae & Great egret & $\begin{array}{c}\text { Ardea alba } \\
\text { (Linnaeus,1758) }\end{array}$ & $\begin{array}{l}\text { Thulo seto } \\
\text { bakulla }\end{array}$ & $\mathrm{R}$ & $\mathrm{C}$ & LC & W1 \\
\hline 40 & Ardeidae & Cattle egret & $\begin{array}{c}\text { Bubulcus ibis } \\
\text { (Linnaeus, 1758) }\end{array}$ & $\begin{array}{l}\text { Bastu } \\
\text { bakulla }\end{array}$ & $\mathrm{R}$ & $\mathrm{C}$ & LC & W1 \\
\hline 41 & Ardeidae & $\begin{array}{l}\text { Intermediate } \\
\text { Egret }\end{array}$ & $\begin{array}{l}\text { Ardea intermedia } \\
\text { (Wagler, 1827) }\end{array}$ & $\begin{array}{l}\text { Sano } \\
\text { bakulla }\end{array}$ & $\mathrm{R}$ & $\mathrm{C}$ & LC & $\mathrm{W} 1$ \\
\hline 42 & $\begin{array}{l}\text { Threskiornithi } \\
\text { dae }\end{array}$ & Black ibis & $\begin{array}{c}\text { Pseudibus papillosa } \\
\text { (Temminck,1824) }\end{array}$ & $\begin{array}{l}\text { Karra } \\
\text { sawari }\end{array}$ & WV & $\mathrm{O}$ & LC & W2 \\
\hline \multicolumn{9}{|c|}{ PICIFORMES } \\
\hline 43 & Megalaimidae & $\begin{array}{l}\text { Blue-throated } \\
\text { Barbet }\end{array}$ & $\begin{array}{c}\text { Megalaima asiatica } \\
\text { (Latham, 1790) }\end{array}$ & Kuthukre & $\mathrm{R}$ & $\mathrm{O}$ & LC & $\mathrm{F} 1$ \\
\hline 44 & Picidae & $\begin{array}{l}\text { Fulvous-brested } \\
\text { woodpecker }\end{array}$ & $\begin{array}{c}\text { Dendrocopos macei } \\
\text { (Vieillot, 1818) }\end{array}$ & Kastha kut & $\mathrm{R}$ & $\mathrm{C}$ & $\mathrm{LC}$ & $\mathrm{F} 2$ \\
\hline \multicolumn{9}{|c|}{ PSITTACIFORMES } \\
\hline 45 & Psittacidae & $\begin{array}{l}\text { Rose-ringed } \\
\text { Parakeet }\end{array}$ & $\begin{array}{c}\text { Psittacula krameri } \\
\text { (Scopoli, 1769) }\end{array}$ & $\begin{array}{l}\text { Kanthe } \\
\text { suga }\end{array}$ & $\mathrm{R}$ & $\mathrm{C}$ & LC & $\mathrm{F} 2$ \\
\hline \multicolumn{9}{|c|}{ STRIGIFORMES } \\
\hline 46 & Strigidae & Spotted Owlet & $\begin{array}{c}\text { Athene brama } \\
\text { (Temminck, 1821) }\end{array}$ & Laatkosero & $\mathrm{R}$ & $\mathrm{C}$ & LC & $\mathrm{F} 2$ \\
\hline 47 & Strigidae & Jungle Owlet & $\begin{array}{c}\text { Glaucidium } \\
\text { radiatum } \\
\text { (Tickell, 1833) }\end{array}$ & Dundul & $\mathrm{R}$ & $\mathrm{C}$ & $\mathrm{LC}$ & $\mathrm{F} 1$ \\
\hline 48 & Strigidae & $\begin{array}{l}\text { Brawn Hawk } \\
\text { Owl }\end{array}$ & $\begin{array}{l}\text { Ninox scutulata } \\
\text { (Raffles, 1822) }\end{array}$ & $\begin{array}{l}\text { Kaal } \\
\text { pechak }\end{array}$ & $\mathrm{R}$ & $\mathrm{O}$ & LC & $\mathrm{F} 2$ \\
\hline \multicolumn{9}{|c|}{ SULIFORMES } \\
\hline 49 & $\begin{array}{l}\text { Phalacrocoraci } \\
\text { dae }\end{array}$ & Little Cormorant & $\begin{array}{c}\text { Phalacrocorax niger } \\
\text { (Vieillot, 1817) }\end{array}$ & $\begin{array}{l}\text { Saano } \\
\text { jalewa }\end{array}$ & WV & FC & LC & $\mathrm{W} 1$ \\
\hline
\end{tabular}

$\mathrm{SS}=$ Seasonal Status, $\mathrm{AB}=$ Abundance, $\mathrm{R}=$ Resident, $\mathrm{WV}=$ Winter Visitor, $\mathrm{SV}=$ Summer Visitor, $\mathrm{C}=$ Common, $\mathrm{FC}=$ Fairly Common, $\mathrm{O}=$ Occasional, $\mathrm{UC}=$ Uncommon, ${ }^{\mathrm{a}}$ Near threatened, ${ }^{\mathrm{b}}$ Vulnerable, 
IUCN= IUCN status of threatened species 2018, LC = Least concern, VU = Vulnerable, HL= Habitat location, F1= Forest area (west), F2= Forest area (east), W1= Wetland area (East), W2= Wetland area (West), HL= Habitat location.

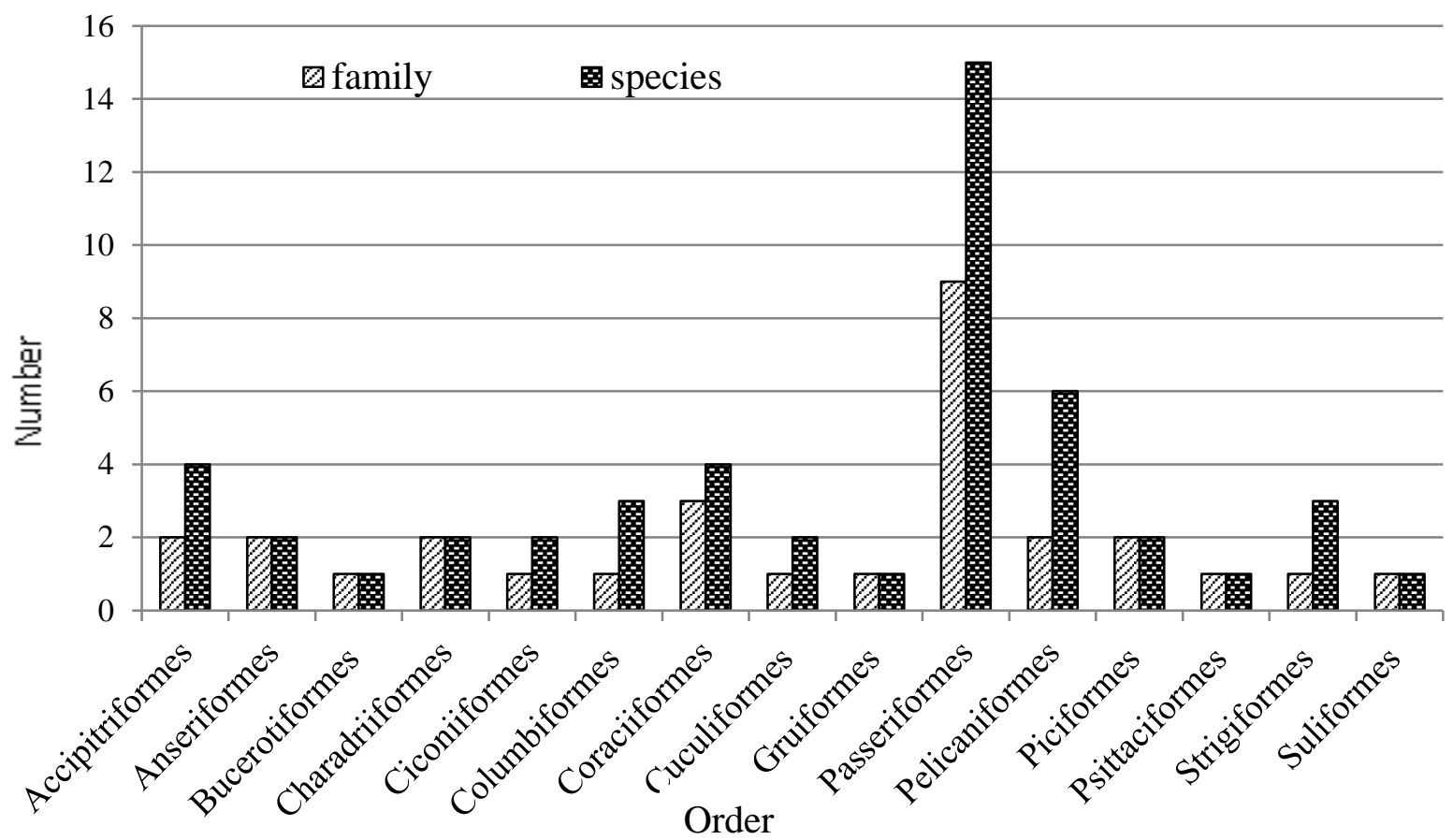

Fig. 2: Avifaunal composition of Betana wetland (order-wise).

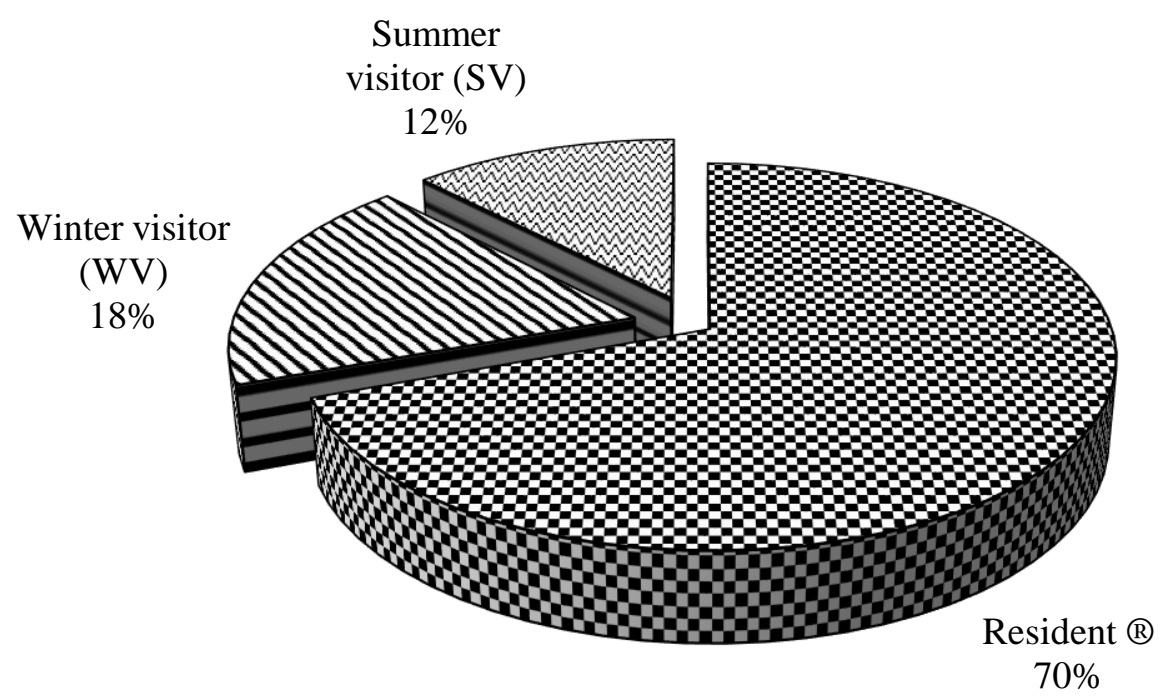

Fig 3: Pie showing seasonal status of avifauna on percentage basis. 


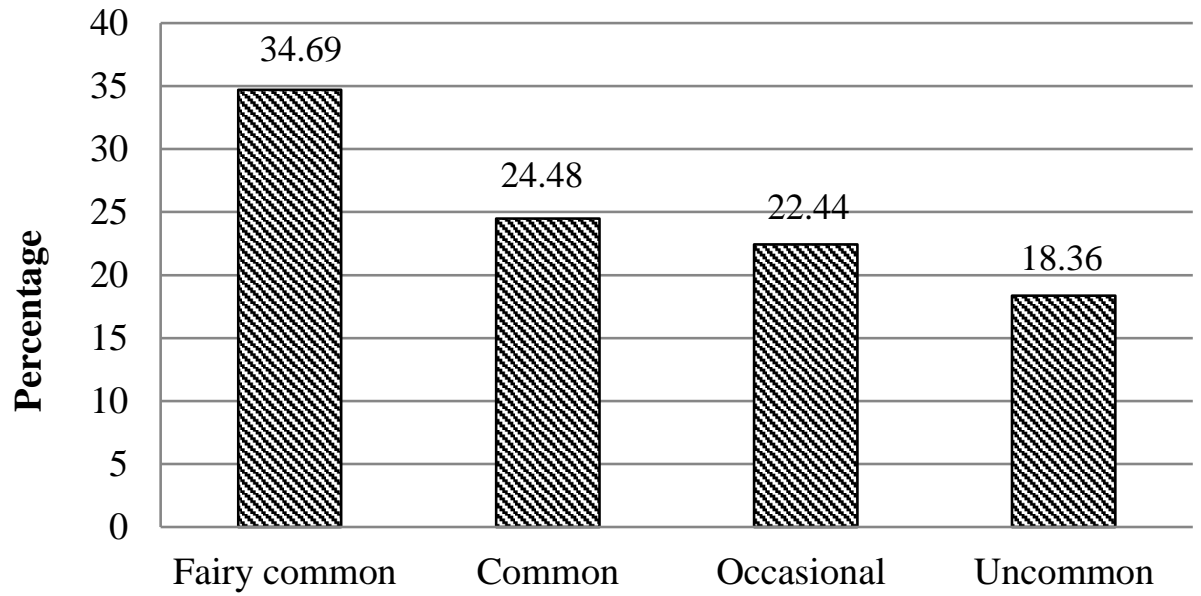

Fig 4: Species dispersal of avifauna.

Table 2: Habitat wise composition of avifauna.

\begin{tabular}{|l|l|l|l|l|l|l|l|}
\hline Habitat location & $\mathbf{N}$ & $\mathbf{\%}$ & $\mathbf{n}$ & $\mathbf{\%}$ & $\mathbf{S V}$ & $\mathbf{W V}$ & $\mathbf{R}$ \\
\hline Wetland area W1 & 10 & 20.40 & 89 & 27.81 & 01 & 03 & 06 \\
\hline Wetland area W2 & 06 & 12.24 & 43 & 13.43 & -- & 01 & 05 \\
\hline Forest area F1 & 16 & 32.65 & 112 & 35.00 & 01 & 01 & 14 \\
\hline Forest area F2 & 17 & 34.69 & 76 & 23.75 & 04 & 04 & 09 \\
\hline Total & $\mathbf{4 9}$ & & $\mathbf{3 2 0}$ & & $\mathbf{0 6}$ & $\mathbf{0 9}$ & $\mathbf{3 4}$ \\
\hline
\end{tabular}

Source: Field survey, 2018. ( $N=$ Number of species, $n=$ Number of individuals, $S V=$ Summer visitor, $\mathrm{WV}=$ Winter visitor, $\mathrm{R}=$ Resident).

Table 3: Shannon Weiner diversity index $(\mathrm{H})$ and Species evenness (E).

\begin{tabular}{|c|c|c|c|c|c|c|}
\hline $\begin{array}{l}\text { Sampling } \\
\text { stations }\end{array}$ & $\begin{array}{l}\text { No. of } \\
\text { individuals } \\
\text { (n) }\end{array}$ & $\begin{array}{l}\mathbf{P i} \\
(=\mathbf{n} / \mathbf{N})\end{array}$ & $\ln \mathbf{P i}$ & $\mathbf{P i}^{*} \ln \mathbf{P i}$ & $\begin{array}{c}\mathbf{H} \\
\left(=-\sum p \mathbf{i}^{*} \ln \mathrm{Pi}\right)\end{array}$ & $\begin{array}{c}E \\
(=H / \operatorname{logS})\end{array}$ \\
\hline W1 & 89 & 0.278125 & -1.27968 & -0.35591 & \multirow{5}{*}{1.33448} & \multirow{5}{*}{$\begin{array}{c}\mathbf{0 . 7 8 9} \\
(\text { for } S=49)\end{array}$} \\
\hline W2 & 43 & 0.134375 & -2.00712 & -0.26971 & & \\
\hline F1 & 112 & 0.35 & -1.04982 & -0.36744 & & \\
\hline $\mathrm{F} 2$ & 76 & 0.2375 & -1.43759 & -0.34143 & & \\
\hline Total & $\mathrm{N}=\mathbf{3 2 0}$ & $\sum \mathbf{P i}=1$ & $\begin{array}{l}\sum \ln P i= \\
-5.77422\end{array}$ & $\begin{array}{l}\sum \mathrm{Pi}^{*} \ln \mathrm{Pi}= \\
-1.33448\end{array}$ & & \\
\hline
\end{tabular}




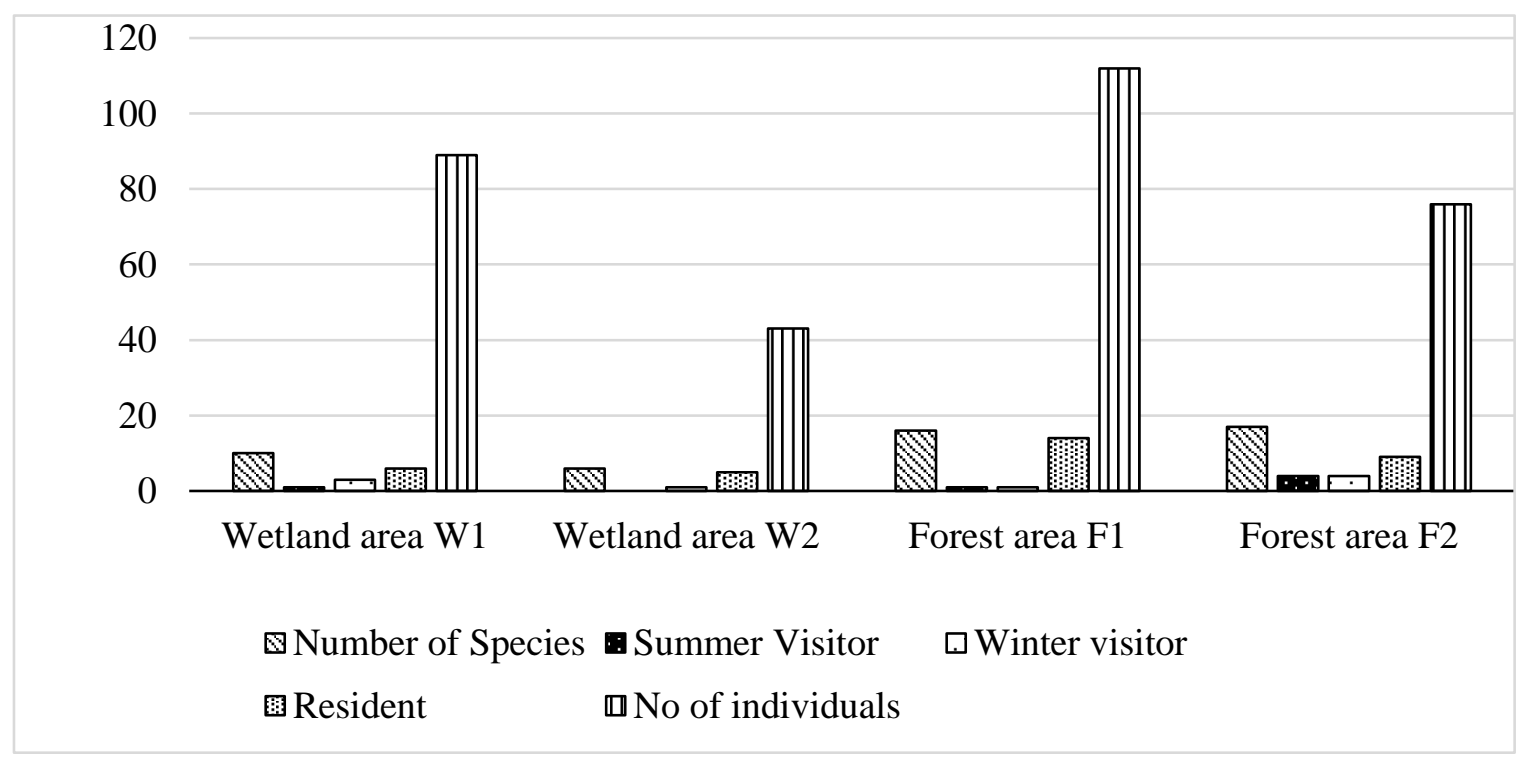

Fig 5: Habitat wise composition of birds.

\section{Discussion}

In the present study most of the birds were found belong to the order Passeriformes including nine families and 15 species, followed by Coraciformes with three families and four species then Pelicaniformes with two families and six species. Of them, two species Grey-headed fish eagle (Icthyophaga humilis) and Lesser Adjutant Stork (Leptoptilos javanicus ) are kept under near threatened (NT) and vulnerable (VU) categories of IUCN Red List status of threatened species respectively [13]. Surana [14] reported 109 species of birds from Chimdi lake. They belong to 34 families where 28 species were resident, 27 species were winter visitor, 17 species were summer visitor 37 species were migratory. Of them, 16 species were common, 23 species were fairly common, 25 species were occasional and 45 species were scarce. Pokharel [15] recorded 295 individuals belonging to 55 species of birds from 26 families and 10 orders from the Betana wetland area but in the present study, altogether 320 individuals belonging to 49 species of birds with 30 families and 15 orders were recorded. Black Kite (Milvus migrans), Indian Roller (Coracius benghalensis) and Whitethroated Kingfisher (Halcyon smyrnensis) as very common species in the Betana wetland. But this time these species were occasionally seen. This may be due to limitation of survey period or due to shortage of food resources (?). However, Red-vented Bulbul (Pycnonotus cafer), Rose-ringed Parakeet (Psittacula krameri), Black Drongo (Dicrurus macrocercus), Oriental Magpie Robin (Copsychuss aularis), Common Myna (Acridotherestristis), Great Egret (Casmerodius albus), House Sparrow (Passer domesticus), Spotted Dove (Streptopelia chinensis) etc. were recorded as high population especially in winter season. In the previous study, Out of 55 species, 44 species were resident, five species were winter visitor and six species were summer visitor but this time, out of 49 species, 34 species were resident $(70 \%)$, nine species $(18 \%)$ were winter visitor and six species $(12 \%)$ were summer visitor. Four more species of winter visitor birds were recorded this time. The maximum number of summer visitor $(8.16 \%)$ and winter visitor $(8.16 \%)$ were recorded from sampling station F2 forest area while maximum number of resident types of birds (28.57\%) were recorded from the sampling stations $\mathrm{F} 1$ forest area. The total number species of birds were recorded from the both sampling areas of forest F1 and F2 was $67.34 \%(n=33)$ and that of from the both sampling areas of wetland W1 and W2 was 32.64\% ( $\mathrm{n}=16)$. Pokharel (2015) calculated Shannon - 
Weiner diversity index and evenness index 1.27 and 0.82 respectively. In the present study, it was found to be 1.332 and 0.789 respectively. This indicates the study area now has higher diversity and species evenness.

\section{Conclusions}

Compared to the previous work, birds belonging to four more families and five more orders were recorded this time. However, total numbers of species were recorded less during the present study. The species richness was found higher in winter season than summer. This may be due to easy availability of food, suitable climate, temperature and migration of species. During the present survey, 320 individuals belonging to 49 species of birds under 30 families and 15 orders have been recorded from the Betana wetland, which proves that the study area is one of the suitable habitats for avifaunal abundance. About $70 \%$ of total bird recorded was found to be the resident type and about $35 \%$ of total recorded bird species were found common. The maximum species of birds were recorded from the forest area $67.34 \%(n=33)$ where the maximum number of individuals were found $35 \%(n=112)$. Of them maximum number of species $69.38 \%(n=34)$ species of birds were resident type. The major threats to the avifauna in the study site were found to be deforestation, overgrazing, bird killing by using catapult and pollution due to recreational activities.

\section{Acknowledgements}

I am thankful to the local people who helped during field visits. I would like to acknowledge the Head, Department of Zoology, Central Campus of Technology, Dharan (TU) for providing the concerned literatures. Thanks are due to IDEA WILD ORG., USA for donating field gears.

\section{References}

[1] R. Grimmet, C. Inskipp, T. Inskipp, Birds of Nepal. Helm Field Guide. Prakash Books, New Delhi. 2000.

[2] Birdlife Nepal, http://www.birdlifenepal.org/status-of-birds.php Accessed $20^{\text {th }}$ Aug 2018.

[3] T. K. Shrestha, Birds of Nepal. Field ecology, J. Nat. His. and Con. 1 (2000) 287.

[4] G. E. Hollis, M. M. Holland, J. S. Larson, Wise use of wetlands, J. Nature and Resource 24 (1998) 213 .

doi: http://dx.doi.org/10.3126/on.v2i1.323

[5] B. R. Subba, D. C. Chhetri, Study of fauna of Betana village development committee, Morang, Nepal. (2005), A report submitted to APEC, Biratnagar.

[6] S. K. Rai, Algal flora of Betana wetland, Morang, Nepal. J. Nepalese Journal of Biosciences 1 (2011) 104-113. doi: http://dx.doi.org/10.3126/njbs.v1i0.7477

[7] Webpage https://en.climate-data.org/location/47719/

[8] R. L. Hutto, S. M. Pletscher, P. Hendricks, A fixed- radius point count method for nonbreeding and breeding season use. J. Auk 103 (1986) 593-602.

[9] S. Ali, S. D. Ripley, Handbook of the Birds of India and Pakistan, Vol. V, Oxford University Press. 1986. doi: https://www.jstor.org/stable/4086054 / 10.2307/4086054

[10] R. L (Sr) Fleming, R. L. (Jr.) Fleming, L.S. Bangdel, Birds of Nepal with reference to Kashmir and Sikkim. First Adarsh Impression, Gaurav Offset, Delhi. 2000.

[11] R. Grimmet, C. Inskipp, T. Inskipp, H. S. Baral, Birds of Nepal, Helm Field Guide. Revised edition, Christopher Helm, London. 2016. doi: https://doi.org/10.1017/S0959270901240170.

[12] P. Pradhan, A pocket photo guide to the birds of Nepal. Falcon Publication, Singapore. 2016.

[13] Webpage https://www.iucnredlist.org. Accessed on 20 August 2018.

[14] R. Surana, B.R Subba, K. P. Limbu, Avian diversity during rehabilitation stage of Chimdi Lake, Sunsari, Nepal. Our Nature 5 (2007) 75-80.

doi: http://dx.doi.org/10.3126/on.v5i1.802.

[15] U. Pokharel, Diversity and conservation status of birds of Betana wetland area, Belbari, Morang, Nepal. M.Sc. Unpublished Thesis. Central Department of Zoology, Tribhuvan University, Kathmandu, Nepal. 2015. 